\title{
MECHANICAL SLIT MASK MECHANISM FOR THE JAMES WEBB SPACE TELESCOPE SPECTROMETER
}

\author{
Simon HENEIN, Peter SPANOUDAKIS, Philippe SCHWAB, Laurent GIRIENS, \\ Leszek LISOWSKI, Emmanuel ONILLON, Leif I. MYKLEBUST \\ Centre Suisse d'Electronique et de Microtechnique (CSEM), Jaquet-Droz 1, CH-2007 Neuchâtel, Switzerland. \\ simon.henein@csem.ch.+41327205111
}

\begin{abstract}
This paper presents the mechanical slit mask designed for the Near Infrared Spectrograph of the James Webb Space Telescope. This mechanism is designed to function at a cryogenic temperature of $30 \mathrm{~K}$, in vacuum. The reconfigurable mask allows to form 24 optical slits in a $137 \times 137 \mathrm{~mm}^{2}$ field of view. The slit length is fixed $(4.8 \mathrm{~mm})$ and their width can range from $50 \mu \mathrm{m}$ to $137 \mathrm{~mm}$. The slit positioning accuracy is $\pm 5 \mu \mathrm{m}$ and the slit width accuracy is $\pm 8 \mu \mathrm{m}$. The paper concentrates on the working principle of the mechanism which is based on an improved "inch-worm" stepping motion of 48 masking bars forming the optical curtain. Voice coil actuators are used to drive the various clutches and the principal mobile stage. Ratchets which engage in the teeth of a rack machined on the bars allow to cancel the accumulation of motion errors as steps succeed one another. The design makes significant use flexure structures. Cryogenic performance and life tests have been performed successfully on subassemblies of the mechanism.
\end{abstract}

\section{INTRODUCTION}

The highly successful mission of the Hubble Space Telescope has provided astonishing images to astronomers of the deepest reaches of outer space. The James Webb Space Telescope (JWST) will replace the Hubble when it is decommissioned near the end of this decade. Its development is made by international partners under the responsibility of NASA and the European Space Agency.

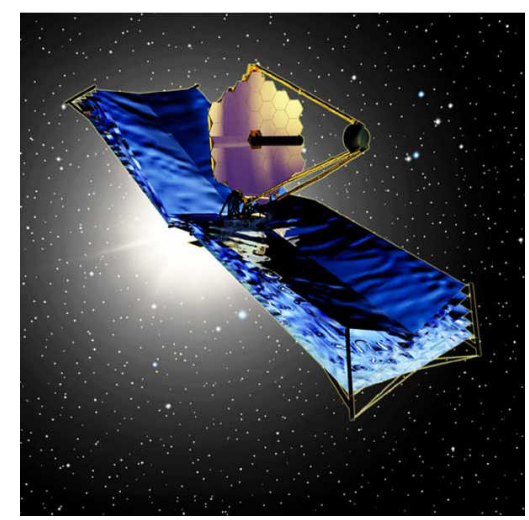

Figure 1: Artist's view of the James Webb Space Telescope
Understanding the origin and evolution of the cosmos critically depends on the astronomer's ability to make quantitative measurements of physical parameters such as total mass, distribution, motions, temperatures, and composition of matter in the Universe. Detailed information on these properties is to be obtained by a highly sensitive Near-Infrared Spectrograph (NIRSPEC), which is one of the three reference science instruments on the JWST. CSEM is responsible for the design, manufacture and test of a Mechanical Slit Mask (MSM). This MSM is a mechanically actuated, reconfigurable slit-mask mechanism (multiple apertures diaphragm) used to select up to 24 celestial objects from the telescope's field of view to be analyzed by the spectrometer. The light coming from the other objects in the field of view is blocked by the mask.

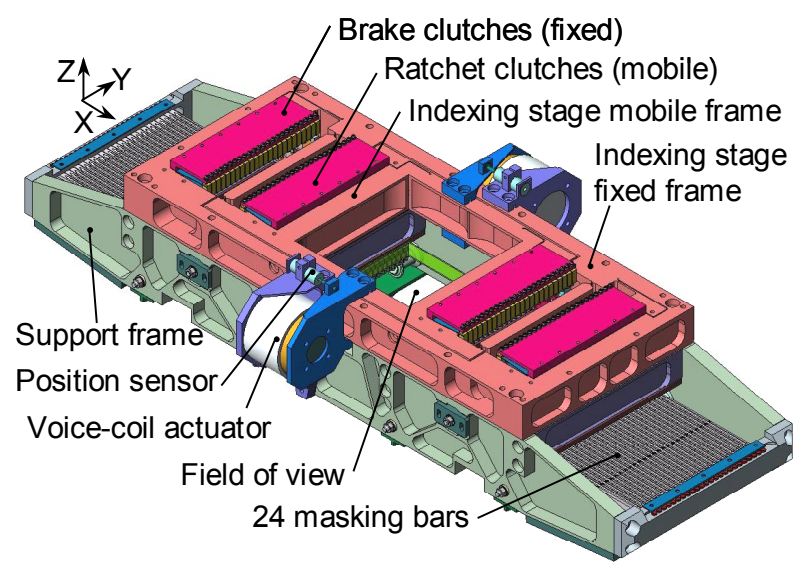

Figure 2: Mechanical Slit Mask Mechanism (MSM)

An object is selected in the field-of-view, by creating a slit that is formed by translating two opposite prismatic bars towards each other on the focal plane of the instrument. The masking bars block all unwanted light except for a small slit defined by the two opposite edges of the pair of bars. Both slit position and size are controlled with a micrometric precision (cf. "Performances" section).

The selected Mechanical Slit Mask (MSM) concept was invented by the authors during a feasibility study. This was followed by the current pre-development phase which consists in designing, manufacturing, assembling 
and testing a breadboard prototype representative of the foreseen flight model of the MSM.

This paper presents the working principle, the key mechanical components, and the results obtained from the room temperature and cryogenic tests on subassemblies of the MSM breadboard. As of writing this paper, the breadboard unit is being assembled and it will be thoroughly tested during summer 2003 .
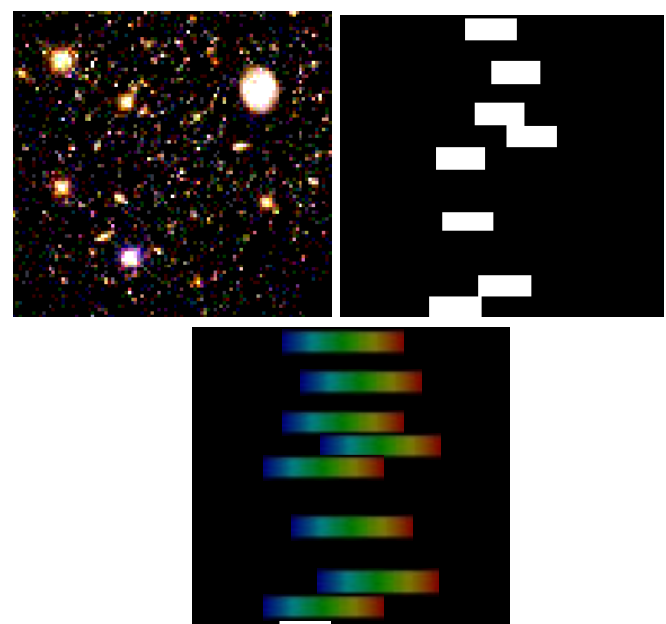

Figure 3: Images in Field Of View (left), slit mask (right) and idealized spectral dispersion of the selected objects (bottom). Only 8 slits (instead of 24) are show on this sketch for clarity.

\subsection{Performances}

The main goal performances of the MSM are :

- Mask field of view area : 137 x $137 \mathrm{~mm}^{2}$

- Number of slits in the mask : 24

- Adjustable slit width from $50 \mu \mathrm{m}$ to $137 \mathrm{~mm}$

- Slit position accuracy: $\pm 5 \mu \mathrm{m}$ over $137 \mathrm{~mm}$ range

- Slit width accuracy: $\pm 8 \mu \mathrm{m}$

- Mask fill factor $92 \%$

- Mask attenuation factor $10^{-8}$

- Withstand launch vibrations and space vacuum

- Cryogenic temperature operation $(20$ - 40K)

- Thermal dissipation: less than $90 \mathrm{~mW}$ (averaged over 3 hours)

- Life time : 5 years in space with 1 full reconfiguration of the mask every 2.5 hours.

- Envelope: 750 x $480 \times 215 \mathrm{~mm}^{3}$

- Mass : $15.8 \mathrm{Kg}$ without launch lock, 23 with launch lock

The thermal dissipation and the vibration survivability have not yet been tested. The other critical performances have been demonstrated experimentally on sub-assemblies of the MSM breadboard (cf. "Cryogenic tests" section).

\section{WORKING PRINCIPLE}

\subsection{Mask curtain (translating bars)}

The mask curtain is composed of two sets of 24 aluminum bars which can translate horizontally across the $137 \times 137 \mathrm{~mm}^{2}$ field of view. The two sets of bars face one another (one set on each side of the field of view). When two opposite bars are driven close to each other, their two frontal edges form a rectangular optical slit. Adjacent bars do not touch one another mechanically, but their cross section is shaped so that they form an optically opaque curtain (Figure 4). Therefore, when all the bars are positioned in the field of view, they form an opaque mask with 24 rectangular openings (slits). The slit width (dimension in the $\mathrm{X}$ direction) can range from $50 \mu \mathrm{m}$ (opposite bars almost touching one other) to $137 \mathrm{~mm}$ (opposite bars fully retracted out of the field of view). The slit length (dimension in the $\mathrm{Y}$ direction) is fixed to $4.8 \mathrm{~mm}$ : it is defined by the masking bars cross section. Placing several adjacent slits in the same $\mathrm{X}$ position allows to form longer slits if required (in that case, the slit length is a multiple of $4.8 \mathrm{~mm}$ ).

The bars have two $\mathrm{V}$ grooves (one on top, one underneath) in which the guiding wheels roll (Figure 4). The bars have a rack on one side composed of 118 teeth manufactured by electro-discharge machining in which the ratchet clutch engages to translate them.

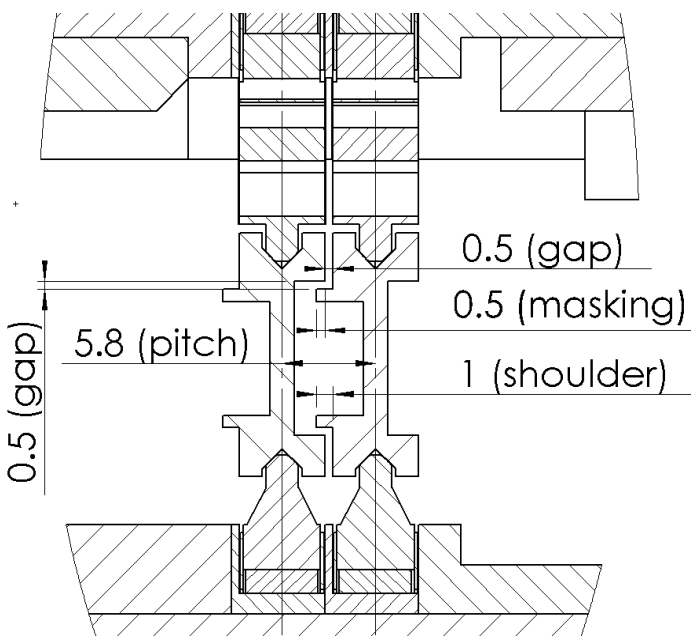

Figure 4 : Masking bars cross section

Two bars are shown on this drawing. Their I shaped cross section leads to a $1 \mathrm{~mm}$ shoulder that blocks the light between adjacent bars. The mechanical gap avoids any contact between the bars. The $V$ grooves machined in the masking bars and the wheels which engage in them are also visible.

\subsection{Bar support wheels and bearings}

The masking bars are guided axially on three titanium wheels which engage in the $\mathrm{V}$ grooves (Figure 5). The wheels are guided on journal bearings (Figure 6). The mechanical play in the bearings is calculated taking into account the thermal shrinkage of the various parts. 
PTFE bushings provide the axial bearing and Vespel bushings provide the radial bearing.

The flexible wheel (Figure 7) is composed of two identical parallel spring stages at 90 degrees with respect to one another. This monolithic design provides a uniform radial stiffness to the wheel, while maintaining a very high stiffness and strength in the other directions.
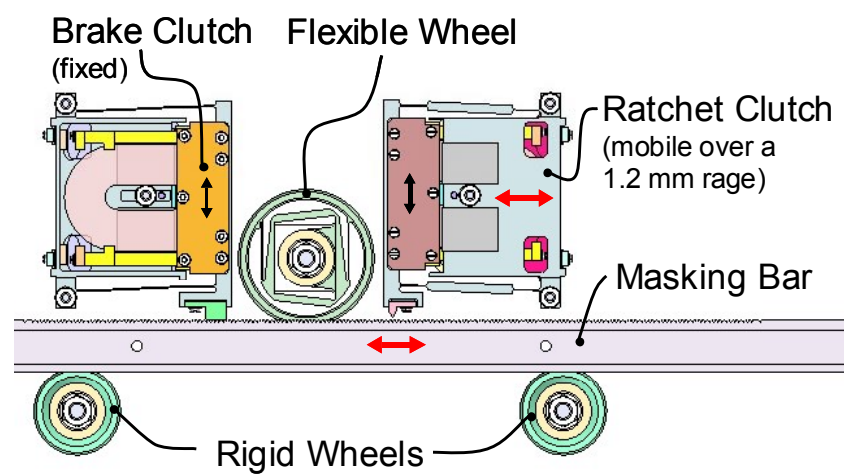

Figure 5: Bar support wheels and bearings The bars are guided on three wheels. The wheels roll in $V$ grooves machined in the bars. One of the wheels is flexible radially. This prevent any over-constraints and allows to control precisely the preload.

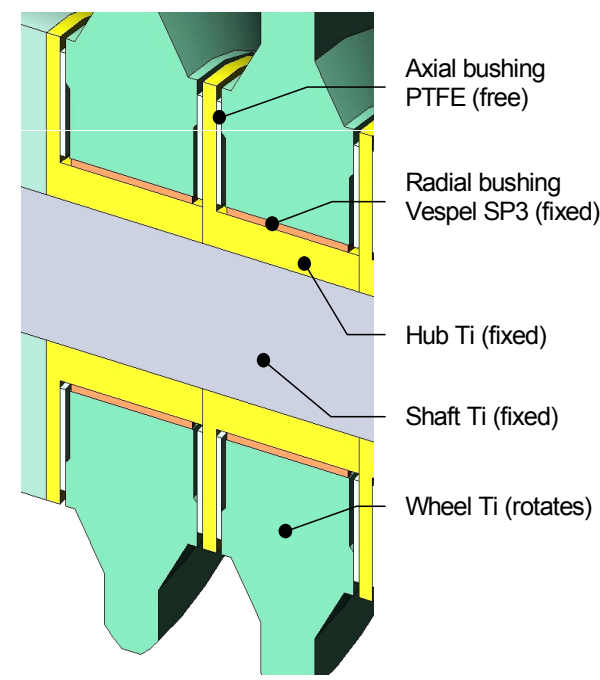

Figure 6: Wheels plain bearings

The plain bearings of the flexible wheel are identical to the one shown in this picture.

The flexible wheel performs two main functions:

- Applying a constant preload force on the masking bar to maintain its contact with the two rigid wheels in space and in all MSM on-ground positions. The worst case position is the upsidedown position, with the bar fully deployed into the field of view. This causes a maximum cantilever effect which tends to lift the bar off the rigid wheel that is the closest to the field of view.
- Accommodating the lift of the bar during the launch clamping phase without creating excessive stresses in the blades.

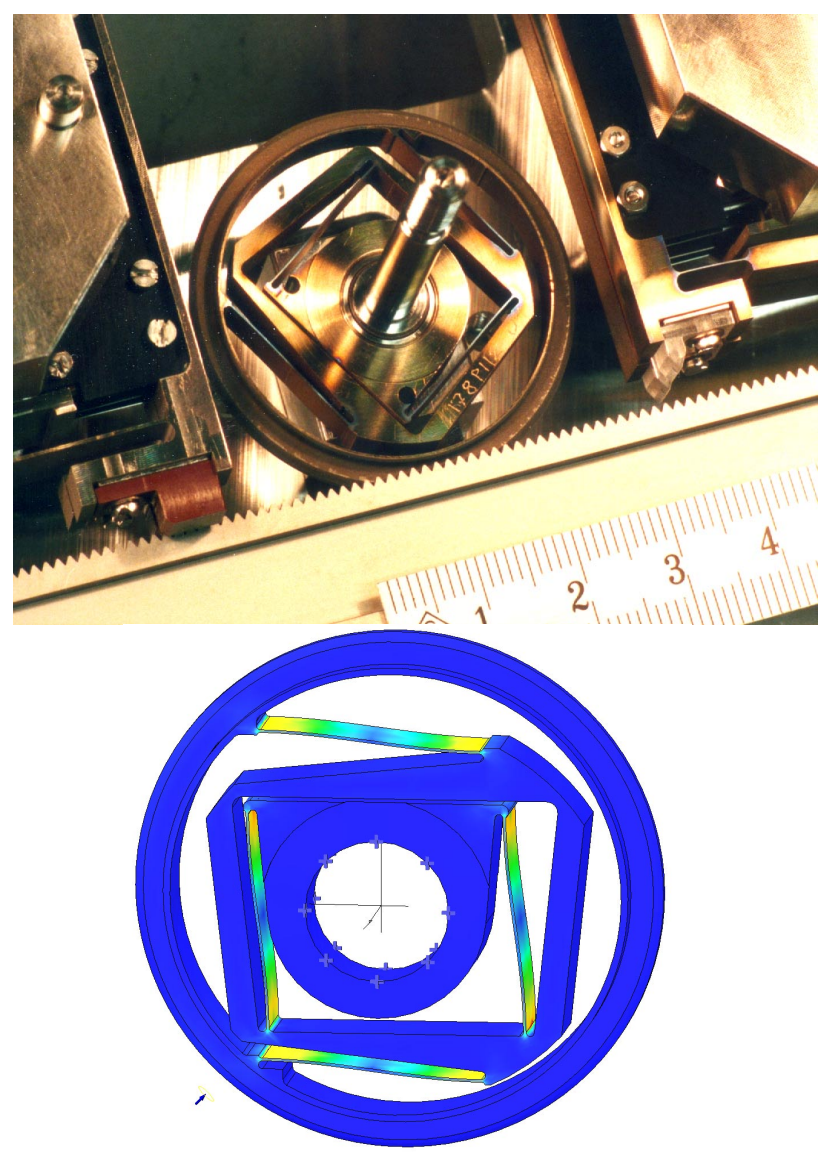

Figure 7 : Flexible Wheel

Picture showing bake pad (left), wheel (center), ratchet (right) and bar (bottom). The centimeter ruler shows the scale. The sketch shows the wheel in a radially deformed position.

\subsection{Bars translation}

\section{Ratchet clutch}

Each bar is translated by a ratchet clutch which can be moved horizontally with very high precision (better than $1 \mu \mathrm{m})$ over a $1.2 \mathrm{~mm}$ range. The clutch is equipped with a sapphire tooth which engages in the corresponding female teeth of the masking bar.

The ratchet clutch is composed of a parallel spring stage with 4 flexures used as a vertical translational bearing. The part is machined pre-deformed, in order to have the flexures straight when the ratchet is engaged in the bar teeth. This arrangement minimizes the parasitic displacement ( $\mathrm{X}$ direction) of the ratchet tooth when it engages in the bar teeth. At rest (actuator powered off) the mobile part of the clutch is pressed against an upper end stop by the elastic restoring force of the bearing. To engage into the masking bar teeth, the ratchet is powered on, which produces a magnetic force. This force moves the sapphire tooth down to the bar. The 
magnetic force overcomes the elastic restoring force of the bearing to press the sapphire tooth in the corresponding female masking bar tooth. The magnetic force is sized to be sufficient to fully engage the ratchet male tooth in the bar female tooth, which centers the bar with respect to the ratchet. The measured engagement repeatability is better than $1 \mu \mathrm{m}$ (cf. "Cryogenic tests" section).

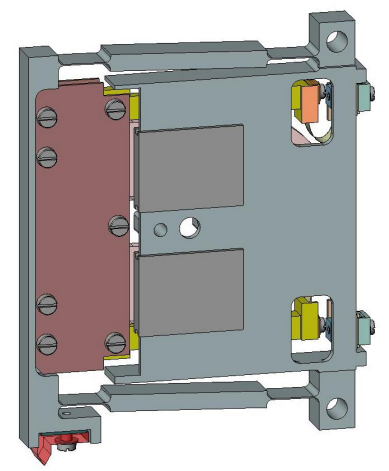

Figure 8 : Ratchet Clutch

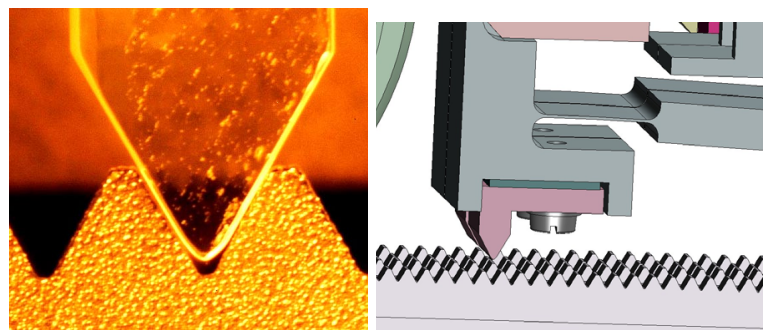

Figure 9 :Picture of the ratchet sapphire tooth engaging into the aluminum masking bar (the distance between two teeth on the masking bar is $1.2 \mathrm{~mm}$ )

\section{Brake clutch}

Once the masking bar has been moved by the ratchet clutch, its position is maintained by a brake clutch ( simply called "brake" further on).

The brake is composed of a parallel spring stage with two flexible blades used as a vertical translational bearing guiding the plastic braking pad, and as a preload spring at the same time. The blades are machined straight, and are then preloaded when the brake is mounted on the MSM mechanism. In micro-gravity, the nominal preload force is sufficient to block the bar without powering the brake actuator. On-ground, in vertical position, the brake actuator has to be powered on with a reverse current during braking phases to increase the braking force and prevent the bar from sliding downwards.

To open the brake, the actuator is powered on, producing an electromagnetic force which overcomes the elastic restoring force. This force lifts the baking pad off the bar.
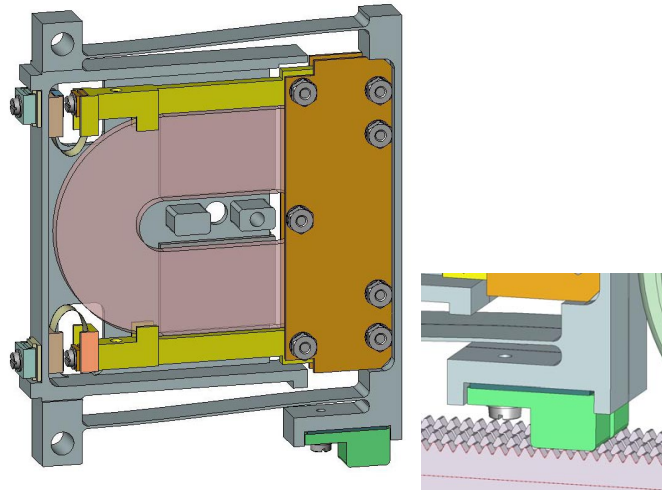

Figure 10: Brake clutch

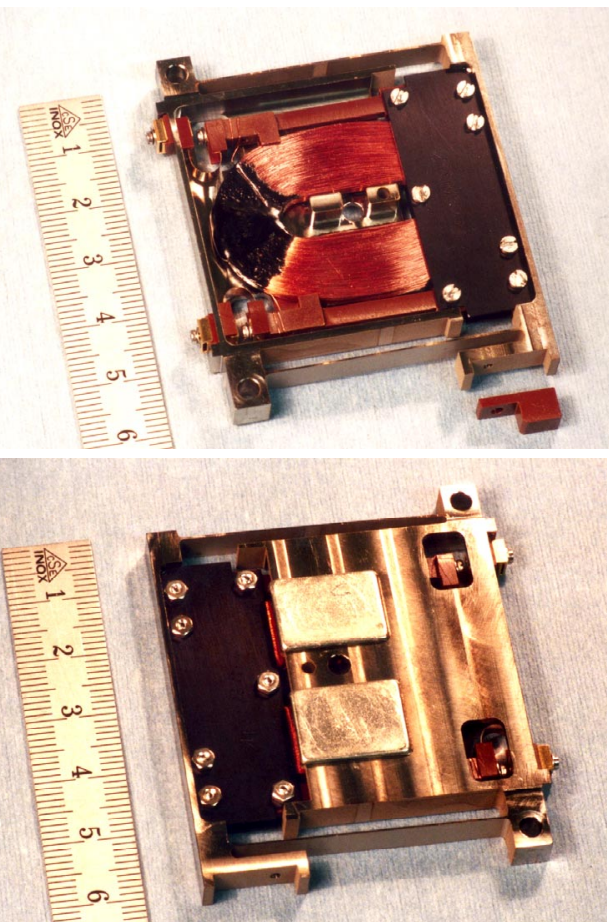

Figure 11: Pictures of the brake clutch (front and back). The centimeter ruler shows the scale.

\section{Clutch actuators : "slice voice-coils"}

The ratchet and brake clutches are based on an innovative electro-magnetic structure. This structure is a sandwich assembly of identical actuator slices stacked to form a single assembly (Figure 12).

Each slice includes a mobile coil and two permanent magnets. The magnetic flux makes a loop going through all the actuator slices. The advantages of such an arrangement are:

- A small thickness of the actuator (5.8 $\mathrm{mm}$ pitch) (no iron needed inside the actuators to guide the flux).

- The magnetic conditions are relatively insensitive to the number of clutches.

- The common flux is closed at the extremities by only two iron yokes. 
This concept is protected by a patent and has been implemented in other applications.

Since the coils are mobile, special electrical connections are required (see "Rolling ribbons section").
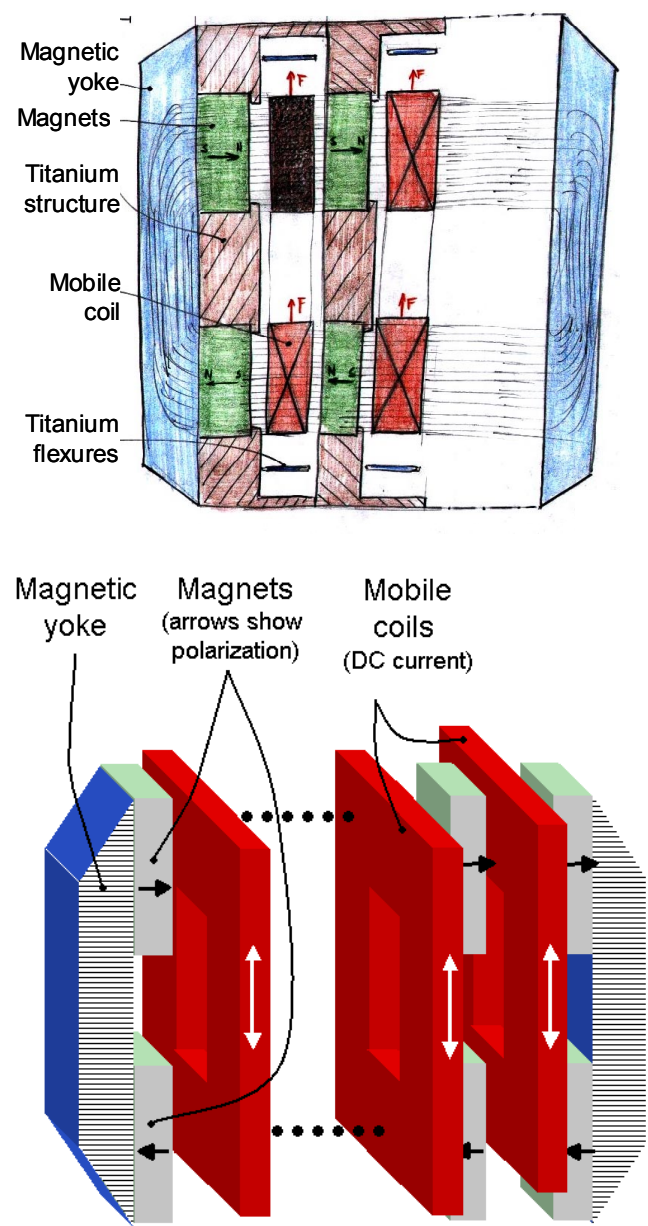

Figure 12:Sketches showing the "slice voice-coil" actuators principle used for the brake and ratchet clutches

\section{Rolling ribbons}

The power is transmitted to the clutch coils (ratchet and brakes have identical actuators) via two thin $(0.015 \mathrm{~mm}$ thickness, $2.8 \mathrm{~mm}$ width) $\mathrm{CuBe}$ ribbons naturally bent between two electrically insulating plates. One of these plates is attached to the fixed base and the other to the mobile coil. The ribbon is rolling frictionless between the two plates that are parallel to the direction of displacement.

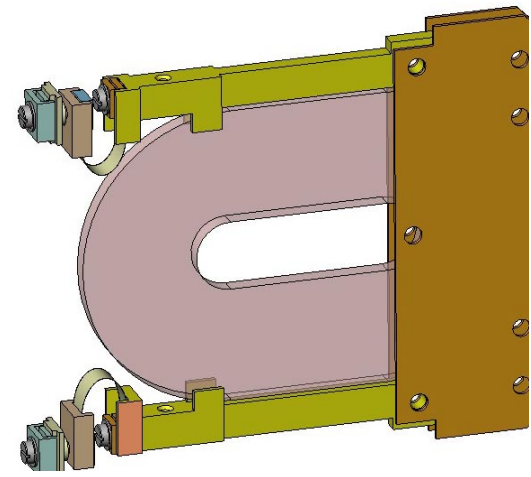

Figure 13: Rolling ribbons

This drawing shows the mobile part of the clutches actuators (coil and mounting plates) with the two rolling ribbons used as electrical connection on the left hand side

\section{$2.4 \quad$ Indexing stage}

The indexing stage (Figure 14) is composed of an outer fixed frame connected to an inner mobile frame by two arms articulated on flexure hinges (flexible necks). The system is machined monolithically by wire-EDM in aluminum. This design leads to a pure translation motion of the mobile frame since the two articulated arms are in parallel and the same length. The translation is not perfectly rectilinear, but follows an arc of circle. The parasitic Y motion is extremely small (less than 1 $\mu \mathrm{m}$ for the $\pm 0.6 \mathrm{~mm}$ nominal stroke) and has no effect on the operation of the device. The two stacks of 24 ratchets are mounted on the two opposite sides of the mobile frame.

The indexing stage is actuated by a voice-coil actuator (Figure 16) (plus a second one for redundancy). This actuator is an off-the-shelf industrial component. The flight model will use a dedicated actuator (with smaller mass and size).

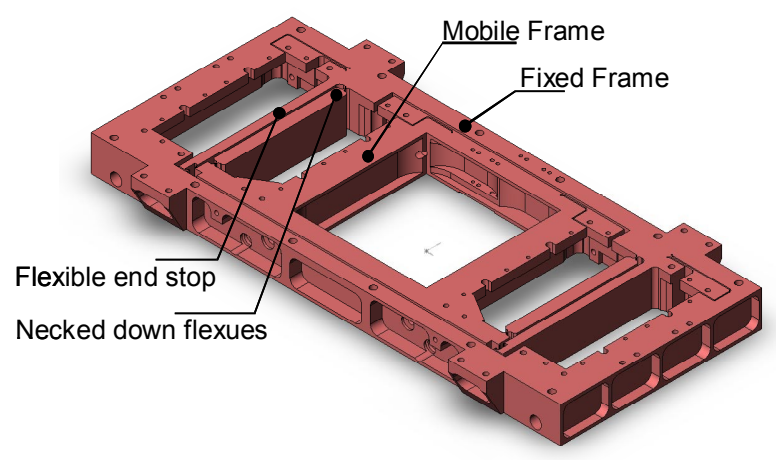

Figure 14: Indexing stage

This monolithic part is machined by EDM in aluminium.

The indexing stage position is measured by an LVDT inductive position sensor with a resolution of $\pm 0.5 \mu \mathrm{m}$. The indexing stage motion is controlled in closed loop using a PID controller. This allows to move the 48 
ratchets all together to any position over a $1.2 \mathrm{~mm}$ range with micrometric precision.

Two mechanical end-stops are integrated in the indexing stage structure. These end-stop consist of flexible cantilever beams which are mechanically preloaded. Hence, the mobile frame can be pressed precisely against the stops (with a force smaller than the preload) for initialization and calibration purposes. In case of accidental shock, the end-stops bend and significantly reduce the shock force preventing any damage on the ratchet assemblies. Two additional hard stops are added to stop the mobile frame in case of overshoots which would surpass the flexible end-stop admissible force (Figure 15).

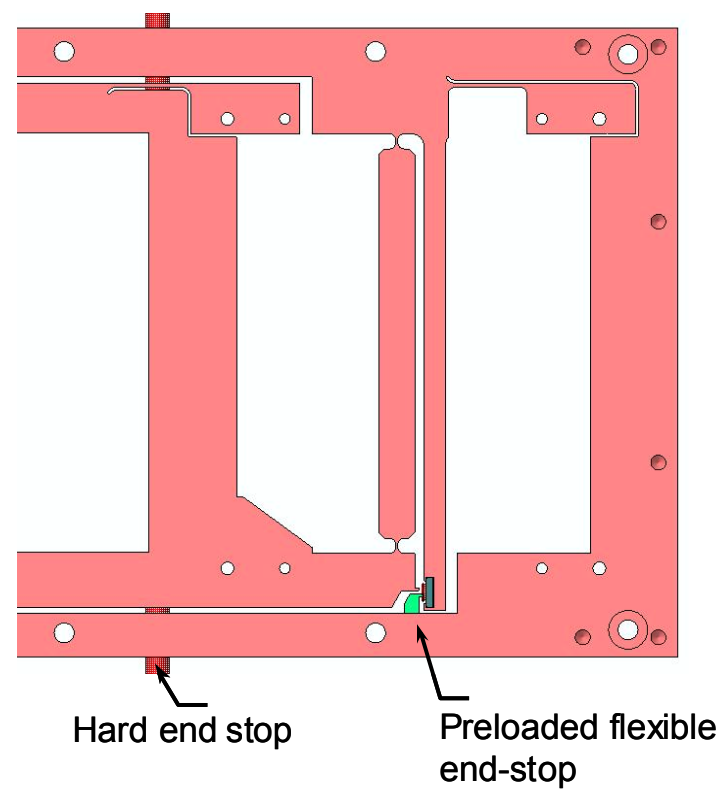

Figure 15: Indexing stage end-stops.

Half the indexing stage is shown on this picture which shows the flexible and hard end-stops used to prevent any overshoot of the mobile frame.

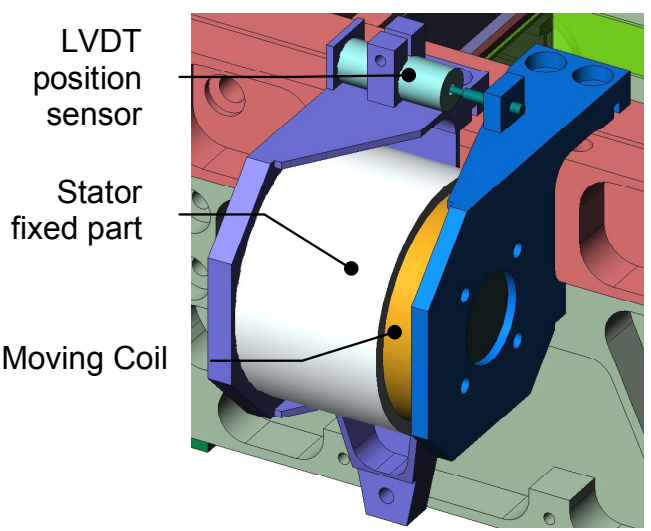

Figure 16: Voice-coil actuator and LVDT position sensor used to control the indexing stage position in closed loop.

\section{MOTION MODES}

Moving a masking bar is done using a simple sequence :

a. move indexing stage to align the corresponding ratchet tooth in front of the masking bar female tooth which is within reach.

b. engage ratchet tooth

c. open brake

d. move the indexing frame to desired position (carrying the masking bar along) over the $1.2 \mathrm{~mm}$ range

e. close brake

f. disengage ratchet

This sequence is repeated sequentially until the goal position is reached.

At the MSM level, two distinct motion modes are used (Figure 17).

\section{Mode 1 : Discrete in-phase group motion}

When all the bars are in-phase (their teeth are aligned), any number of bars (from 1 to 48 ) can be moved one step $(1.2 \mathrm{~mm})$ in any direction at each cycle of the indexing stage. This in-phase motion mode allows to bring the bars quickly to the vicinity of their exact target position. This mode allows to reach only discrete points in the field of view surface (stepping, inch-worm like motion). These points correspond to full $1.2 \mathrm{~mm}$ steps.

\section{Mode 2 : Continuous out-of phase individual motion}

The bars can be moved within single steps only by individual actuation of single bars. This motion can bring the actuated bar anywhere within one step with the precision of the mechanism $( \pm 5 \mu \mathrm{m})$.

Since two opposite bars are used to form a slit, this mode leads to a slit width accuracy of $\pm 8 \mu \mathrm{m}$ (taking into account the statistical distribution of the errors).

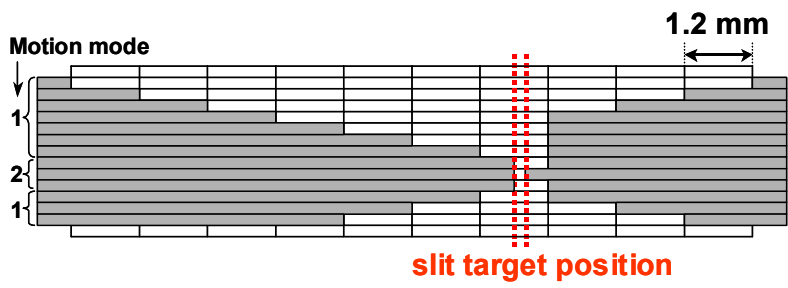

Figure 17: Sequential movement of one pair of bars starting from the edges of the field of view, then moving to the center to form a slit, then moving back towards the fully open position. Each row on this sketch represents the position of a pair of bars after one cyclic motion of the indexing stage which oscillates at a $1 \mathrm{~Hz}$ frequency. In other words, two adjacent rows on this sketch are separated by 1 second. 


\section{LAUNCH LOCKING}

The MSM must withstand the launch environment. The vibration levels are significant for a mechanism with numerous parts and it is necessary to securely clamp the most delicate of them : namely the masking bars, the ratchets, the brakes and the indexing stage. The critical aspect of the components sizing is the induced stresses during launch vibration and the displacements during the locking sequence. These aspects will be validated as part of the breadboard vibration tests.

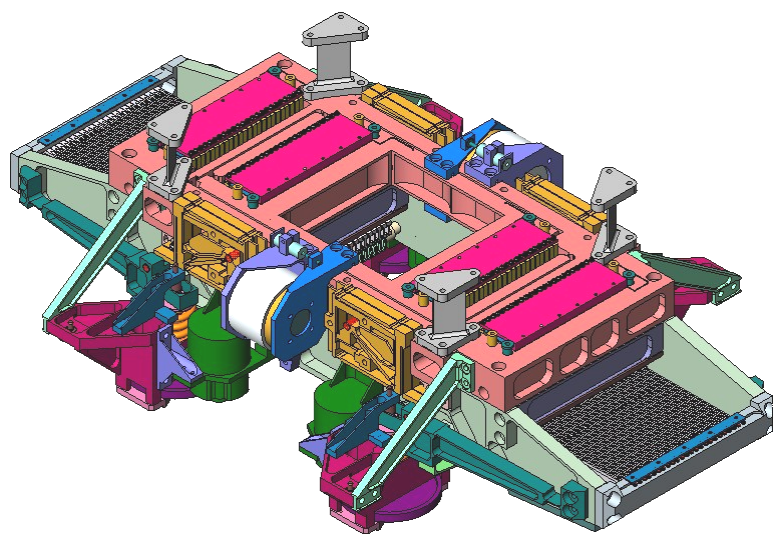

Figure 18: MSM with remotely resetable launch lock

The design guidelines for the locking actuation mechanism were to incorporate redundancy at the highest possible level and avoid single point failures during the launch lock release sequence. The number of actuators was kept to a minimum by using a common actuator to lock masking bars and indexing stage. The masking bar position is set in the retracted configuration (field of view open). The proposed design in remotely resetable, which is necessary during the on-ground test phase which precedes the launch.

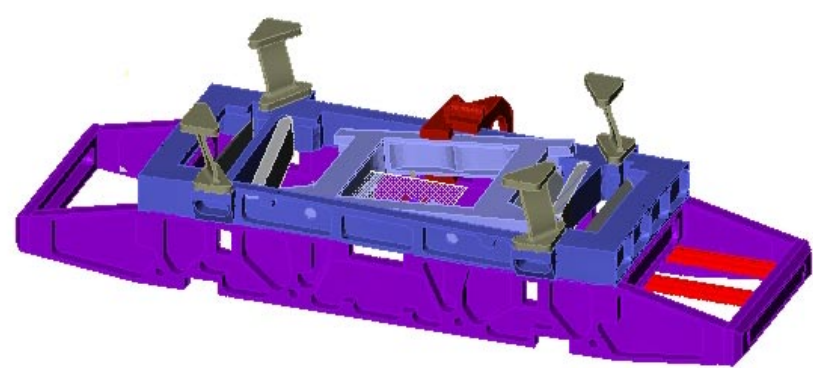

Figure 19: Finite element method analysis of the MSM in locked configuration, showing the first eigen-mode which corresponds to the oscillation of the full mechanism on its 4 interface feet (frequency $140 \mathrm{~Hz}$ )

\section{CRYOGENIC LIFE \& PERFORMANCE TESTS}

The harsh operating environment of extremely low temperatures and high vacuum pose several challenges to cryogenic mechanisms [1] including : out-gassing, physical property changes, differential shrinkage of different materials, tribological modifications, etc.
Cryogenic tests have been performed on MSM subaassemblies at $30 \mathrm{~K}$ and $4.10^{-8} \mathrm{mbar}$ vacuum. The tests covered :

- Ratchet engagements repeatability : better than $1 \mu \mathrm{m}$.

- Accelerated ratchet engagement life cycling : $80^{\prime} 000$ cycles (this number represents 5 years life in space for the aluminum masking bar rack teeth).

- Accelerated masking bars full stroke motions : $12^{\prime} 000$ cycles (this number corresponds to a quarter of the 5 years life in space).

- Masking bar positioning accuracy : better than $5 \mu \mathrm{m}$.

- Friction force measurements validating the theoretical models used for the design and confirming the calculated driving force margins.

Tests on the complete breadboard are planned during summer 2003.

\section{CONCLUSION}

The mechanical solutions selected for the Mechanical Slit Mask Mechanism (MSM) have several original ideas which lead to unique advantages.

The MSM is based on the well known inch-worm like stepping motion used to move objects over long strokes $(137 \mathrm{~mm}$ in the present case) while relying upon a limited stoke actuator $(1.2 \mathrm{~mm})$ [2][3]. With the design presented, this classical principle has been greatly improved by using a ratchet clutch engaging in the masking bars instead of the friction clutches used in the state of the art inch-worm actuators. This original solution allows to align the bar position with respect to the actuator position (i.e. the indexing stage) at each step. In this manner, a position error happening during one step is compensated for during the next step, when the ratchet engages into the bar female tooth and realigns it. Therefore, there is no accumulation of errors as steps succeed one another. This unique feature allows to achieve a bar positioning accuracy of $\pm 5 \mu \mathrm{m}$ over the $137 \mathrm{~mm}$ stroke. This would not be possible with a classical inch-worm actuator relying solely on friction to guarantee the steps length, because of the stepping errors accumulation.

This solution allows to use a single off-the-shelf position sensor, and a single off-the-shelf actuator to control the position of all the 48 masking bars.

Flexible bearings [4] (also called compliant mechanisms or flexures) are used to guide the indexing stage, the 48 ratchets, the 48 brakes and the 48 flexible wheels of the MSM. Numerous other flexible elements are used in the mechanical end-stops, the launch clamping device, the elastic attachments (required to accommodate to the thermal contraction of the different materials), etc. Flexures were selected for their unique advantages: 
absence of friction, high precision, absence of wear, no need for lubrication, high reliability, vacuum and cryogenic compatibility).

The thorough cryogenic experimental tests performed prove the robustness of this complex mechanism which functions as well at room temperature in air, as at $30 \mathrm{~K}$ in $4.10^{-8}$ mbar vacuum.

Vibrations tests have not yet been performed, but finite element simulations show that it is possible to clamp all the mobile parts of the mechanism to survive the launch vibrations and function properly once in space.

The original design presented for the first time in this paper has potential applications not only for space telescopes, but also for ground based telescopes equipped with multi-object spectrometers.

\section{ACKNOWLEDGMENTS}

This project is funded by the European Space Agency. The authors would like to thank B. Henson and L. Scolamiero from ESA; J.-M. Rieubland and L. Dufay from CERN who were responsible for the cryostat where the tests at $30 \mathrm{~K}$ were performed. We also thank S. Bottinelli from Mecartex SA (Losone, $\mathrm{CH}$ ) who supervised the electro-discharge machining of all the flexure elements of the mechanism. We also thank our colleagues from CSEM who participated to the success of this project. I. Kjelberg (management), L. Zago (thermal simulations), R. Ruscony (assembly and cabling), G. Besson (Machining), J.-M Mayor (optical measurements).

\section{REFERENCES}

[1] T. T. Young, J. W. Douglass, K. W. Hodapp, H. Yamada, E. Irvin, L. Robertson, Cryostat mechanism design and fabrication, SPIE 2000, Munich.

[2] D. Henderson, A new inchworm motor for industrial nanopositioning, American Society of Precision Engineering (ASPE) annual meeting, 1995.

[3] C. H. Joshi \& B. R. Bent, Application of Magnetic Smart Materials to Aerospace Motion Control, Proceeding of the 34th Aerospace Mechanisms Symposium, Goddard Space Flight Center, May 10-12, 2000, pp. 137-142.

[4] S. Henein, Conception des guidages flexibles, Collection Meta, Presses Polytechniques et Universitaires Romandes, Lausanne, 2001.
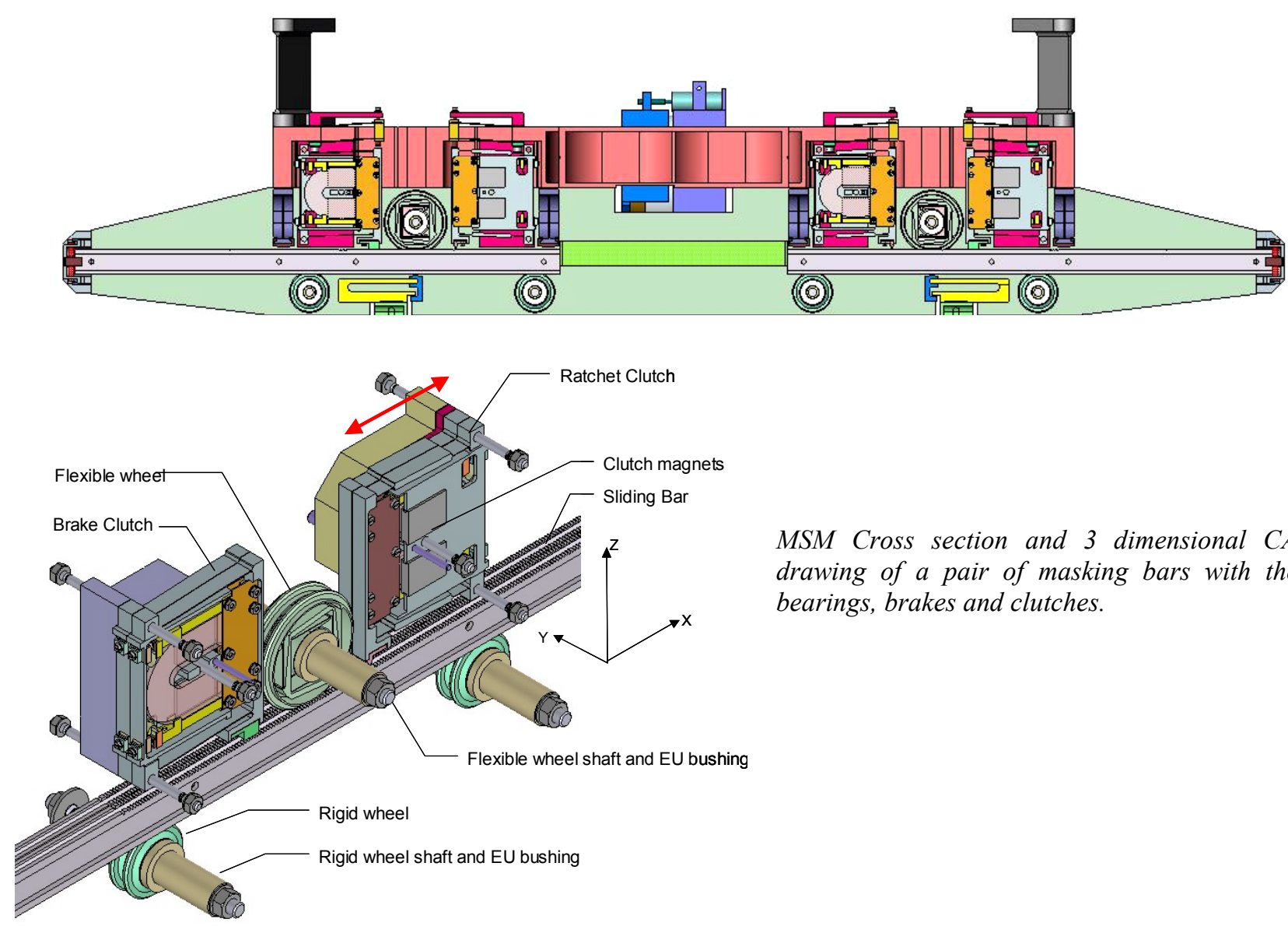

MSM Cross section and 3 dimensional CAD drawing of a pair of masking bars with their bearings, brakes and clutches.

\section{Back to Contents}

